\title{
A Watermarking Technique for Biomedical Images Using SMQT, Otsu, and Fuzzy C-Means
}

\author{
Shaekh Hasan Shoron ${ }^{1}$, Monamy Islam ${ }^{1}$, Jia Uddin ${ }^{1}$, Dongkoo Shon ${ }^{2}$, Kichang Im ${ }^{3}$, \\ Jeong-Ho Park ${ }^{4}$, Dong-Sun Lim ${ }^{4}$, Byungtae Jang ${ }^{4}$ and Jong-Myon Kim ${ }^{2, *(1)}$ \\ 1 Department of Computer Science and Engineering, Brac University, Mohakhali, Dhaka 1212, Bangladesh \\ 2 School of IT Convergence, University of Ulsan, Ulsan 44610, Korea \\ 3 ICT Convergence Safety Research Center, University of Ulsan, Ulsan 44610, Korea \\ 4 Industry IT Convergence Research Group, SW Contents Research Laboratory, Intelligent Robotics Research \\ Division, Electronics and Telecommunications Research Institute (ETRI), Daejeon 34129, Korea \\ * Correspondence: jmkim07@ulsan.ac.kr; Tel.: +82-52259-2217
}

Received: 14 July 2019; Accepted: 29 August 2019; Published: 31 August 2019

check for updates

\begin{abstract}
Digital watermarking is a process of giving security from unauthorized use. To protect the data from any kind of misuse while transferring, digital watermarking is the most popular authentication technique. This paper proposes a novel digital watermarking scheme for biomedical images. In the model, initially, the biomedical image is preprocessed using improved successive mean quantization transform (SMQT) which uses the Otsu's threshold value. In the next phase, the image is segmented using Otsu and Fuzzy c-means. Afterwards, the watermark is embedded in the image using discrete wavelet transform (DWT) and inverse DWT (IDWT). Finally, the watermark is extracted from the biomedical image by executing the inverse operation of the embedding process. Experimental results exhibit that the proposed digital watermarking scheme outperforms the typical models in terms of effectiveness and imperceptibility while maintaining robustness against different attacks by demonstrating a lower bit error rate (BER), a cross-correlation value closer to one, and higher values of structural similarity index measures (SSIM) and peak signal-to-noise ratio (PSNR).
\end{abstract}

Keywords: digital watermarking; biomedical image; successive mean quantization transform; Fuzzy c-means cluster; confidentiality; robustness; imperceptibility

\section{Introduction}

The ongoing advances in technology and computer networks have drawn a significant change in the biomedical sector. Currently, multimedia data are treated as a source for transmitting biomedical information such as patients' reports, hospital information, diagnoses, and so on. This transition encompasses patients, doctors, hospitals, and clinic centers [1]. Among the various types of multimedia data, biomedical images have an immense usage in the biomedical zone. Due to the easy use of the multimedia data, the biomedical images can be easily manipulated during transmission. This initiates huge security issues such as copyright and authentication because the biomedical data are highly confidential. The digital watermarking technique has captured great attention for restricting various illegal hazards and ensuring security. Biometric fingerprints and signatures have been used as the watermarks [2,3]. Due to the sophistication of the medical images, digital watermarking has become more challenging. Although many research works focused on digital watermarking of medical images, there is no single model that adheres to all the security and privacy requirements. Therefore, it is crucial to develop watermarking models to fulfill all the required specifications.

Digital watermarking is a technique of inserting any kind of additional data into the source image. The additional data is known as the watermark. The key feature of watermarking is information 
hiding. Embedding sensitive data in the images facilitates data hiding and ensures maximum privacy. Watermarking medical images has two goals:

i. Controlling reliability and authentication.

ii. Hiding sensitive information of the patient.

Moreover, watermarking procedures can be uniquely classified into three categories based on its function: validation, confidentiality, and a combination of both. During transmission, the image can be distorted due to illegal access, manipulation, and so on. As a result, the image quality is degraded while causing violation of authentication. Digital watermarking provides security as well as maintains the quality of the image. Watermarking ensures several properties of an image such as imperceptibility, robustness, and authentication [3]. Additionally, this process helps to identify the owner of that image.

In this paper, an image watermarking model for protecting biomedical images is proposed. A watermark is embedded into the image using discrete wavelet transform (DWT) and extracted using inverse DWT (IDWT). The image security is ensured by comparing the original watermark before embedding and the extracted watermark after transmission. The following parameters are used to examine the performance of the proposed model: peak signal-to-noise ratio (PSNR), bit error rate (BER), cross correlation (CC), and structural similarity index measures (SSIM).

The rest of this paper is organized as follows. Section 2 shows the literature study related to biomedical image watermarking. Section 3 illustrates the proposed model, which includes the pre-processing, segmentation, embedding, and extracting of the watermark using different algorithms. Section 4 presents the comparison between the proposed model and related models in terms of imperceptibility, robustness, and authentication. Finally, Section 5 concludes this paper.

\section{Related Works}

Several researchers work in the area of digital biomedical image watermarking [4-13]. Most of them use the watermark technique for confidentiality, authentication, and improved security. Heena et al. proposed a model for digital image watermarking using DWT [4]. Rathi et al. proposed a model for an invisible watermarking technique in biomedical images using DWT, discrete cosine transform (DCT), and singular value decomposition (SVD) [5]. Mehto et al. used a combination of DWT and DCT algorithms for embedding and extracting watermarks [6]. Both of the experiments were evaluated using PSNR. Methaq et al. created a method of watermarking using fuzzy image [7]. They embedded a watermark in a fuzzy segmented image. Vikas et al. implemented a method using particle swarm optimization (PSO) and DWT [8], where the host image quality is enhanced using PSO. After that, the watermark is embedded using DWT. Sivadanam et al. proposed a watermarking technique using singular value decomposition (SVD) and stationary wavelet transformation (SWT), where the host image is divided into several bands using SWT and then processed by SVD for embedding the watermark in the image [9]. Mashmood et al. proposed a watermarking algorithm where first the biomedical image is divided into two regions: a region of interest (ROI) and a region of background (ROB) [10]. Then, the watermark is embedded into the ROI because it provides a better result. Eswaraiah et al. [11] and Shukla et al. [12] proposed a model where the input images are divided into two regions: ROI and region of non-interest (RONI). This model focuses on avoiding disturbances by embedding the watermark in the ROI. A different approach was taken by Islam et al. for securing the transmission of watermarked biomedical images [13]. Islam S. and Kim. C. H. have proposed a Hamming code-based error detection using a graphics processing unit (GPU) for accelerating the transmission requirements of a watermarked biomedical image $[4,8]$. Most of the research results are evaluated using PSNR, which shows how robust the image is. Aparna et al. proposed a model for watermarking based on hybridization of compression and cryptography algorithms [14], which consists of the region-growing algorithm and Hash algorithm-256 for embedding and extracting the watermark in the biomedical image. 


\section{Proposed Model}

Biomedical images contain an enormous amount of information. When a watermark is embedded into the image, the information can be distorted. That is why pre-processing and segmentation processing are implemented. The proposed model is illustrated in Figure 1. The detailed description of the model is presented in Subsections 3.1 to 3.3.

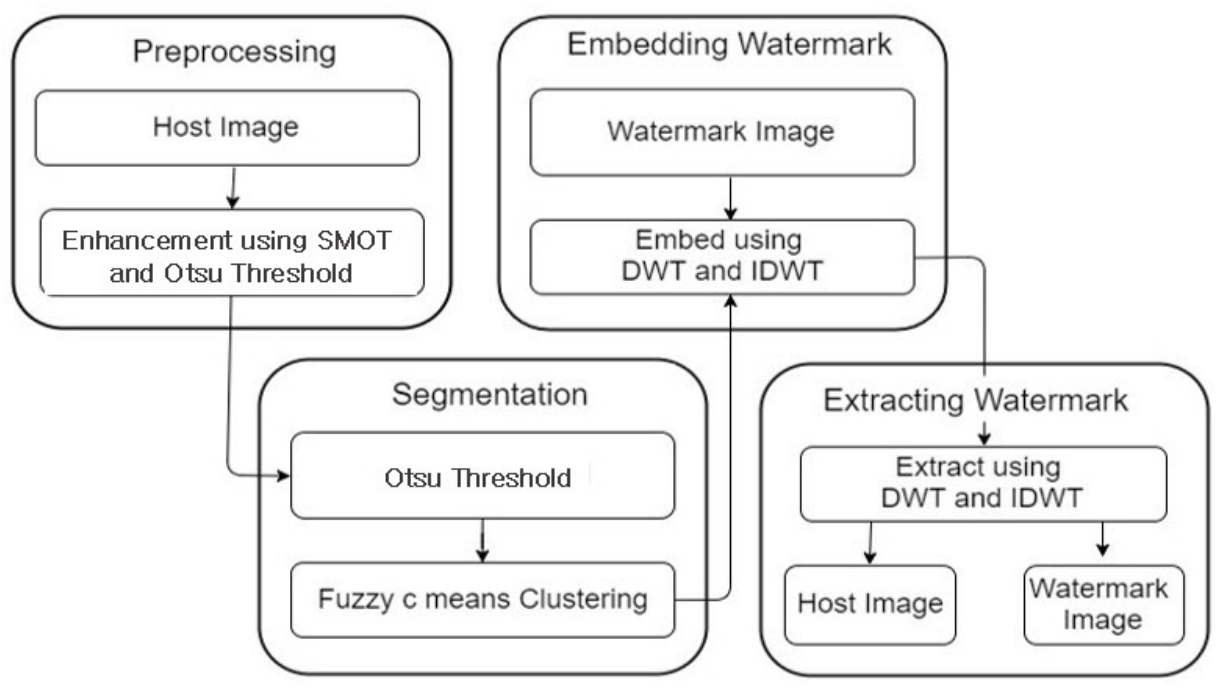

Figure 1. Flowchart of the proposed model.

\subsection{Preprocessing}

Preprocessing provides an added advantage to further analysis. Often during biomedical image transmission, the quality degrades and suffers from blurriness and undesirable noise. To reduce these issues, the sharpness and brightness of the biomedical image was enhanced in the pre-processing stage.

For image enhancement, we used the successive mean quantization transform (SMQT) algorithm with the help of Otsu's threshold. This algorithm converts the image into a binary tree where each node represents the mean quantization unit (MQU). Each of the MQUs works in three steps: mean calculation, quantization, and input set split [15]. In this case, Otsu's threshold value is utilized at the root of the binary tree of the SMQT algorithm. The output tree is shown in Figure 2. The tree is expressed as $\mathrm{u}_{(1, n)}$, where $l$ is the current level and $\mathrm{n}$ is the output number. The final SMQT result is calculated by adding the weighting result of the data points of $\mathrm{u}_{(1, \mathrm{n})}$. The algorithm aims to have a low dynamic image range. That is why the weighting is calculated by $2^{\mathrm{L}-1}$ up to $L=8$.

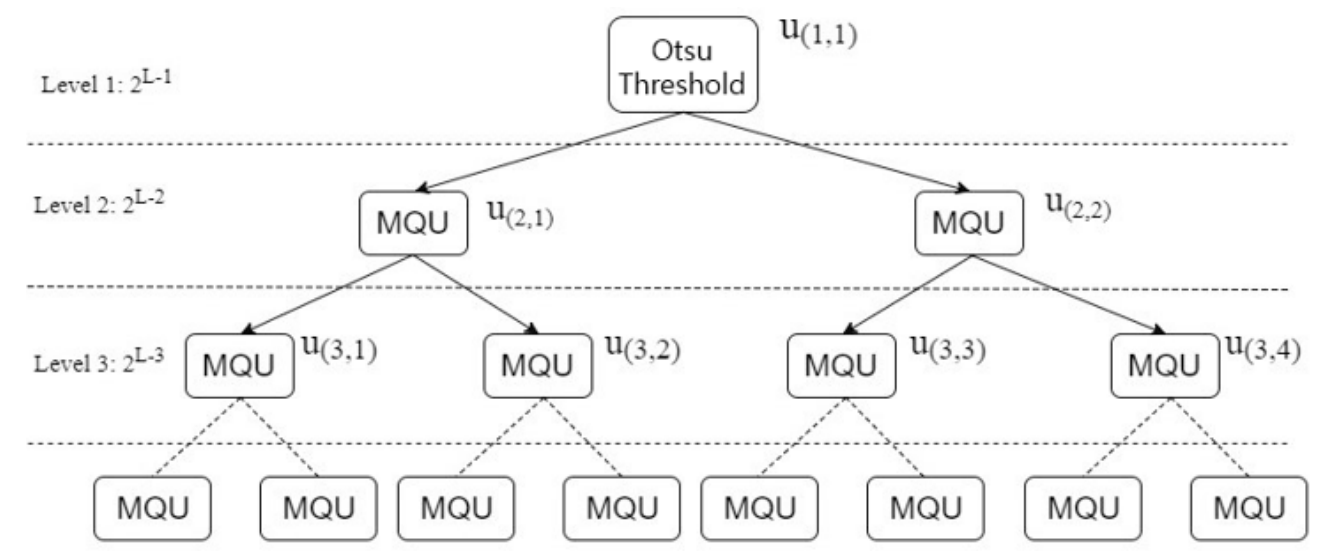

Figure 2. The SMQT as a binary tree of the mean quantization unit (MQU). 


\subsection{Segmentation}

Dividing the image into different sections based on similar traits is called segmentation. Segmentation divides an image into multiple parts, where each part has the same kind of data [16]. For embedding a watermark, image segmentation plays a vital role so that the watermark can be embedded into the correct segment. In the proposed model, we did this segmentation in two steps: the first step is the Otsu's threshold method and the second step is the K-means clustering method. These two steps are described in the Subsections 3.2.1 and 3.2.2.

\subsubsection{Otsu's Threshold}

Otsu's threshold is used in the proposed model because of its constancy and effectiveness [17]. Every image has foreground and background parts. This algorithm finds the threshold value for those positions of the image where foreground and background expansion is minimally possible [18]. Figure 3 shows the preprocessed host image (depicted in Figure 4) and the image after Otsu threshold segmentation.

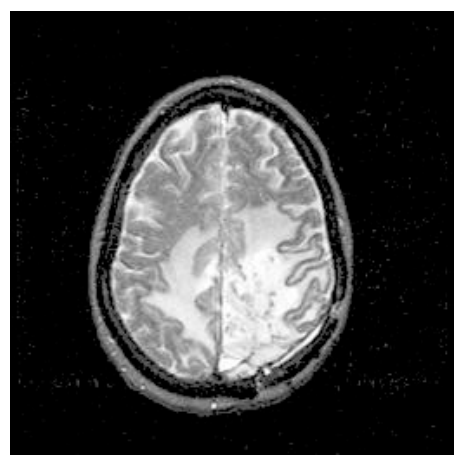

(a)

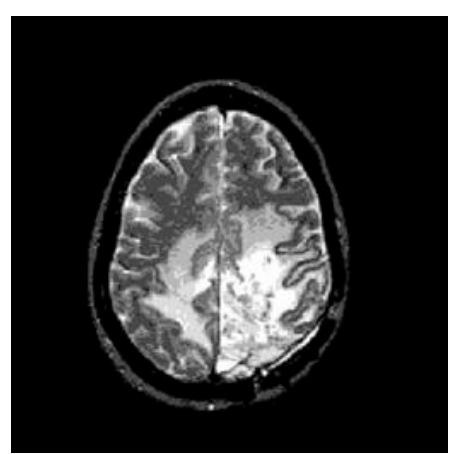

(b)

Figure 3. (a) Preprocessed image, (b) Processed image after Otsu threshold segmentation.

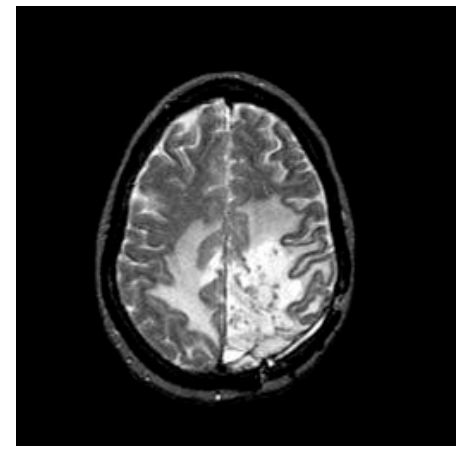

Figure 4. Host Image.

\subsubsection{Fuzzy C-Means Clustering (FCM)}

We used a clustering method for better image segmentation. In the clustering method, different data points of an image are assigned to a group, which is called a cluster. Data points that are in the same cluster have similar kinds of features [19]. FCM algorithm [20] is used in the proposed model for clustering due to its efficiency. Figure 5 demonstrates the host images before and after the Fuzzy c-means clustering. 


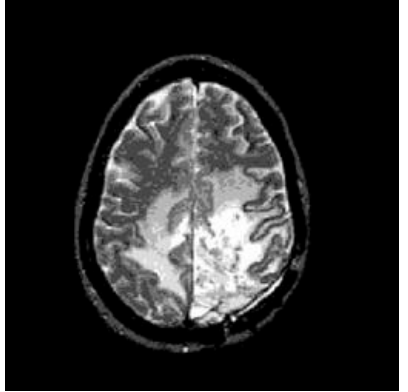

(a)

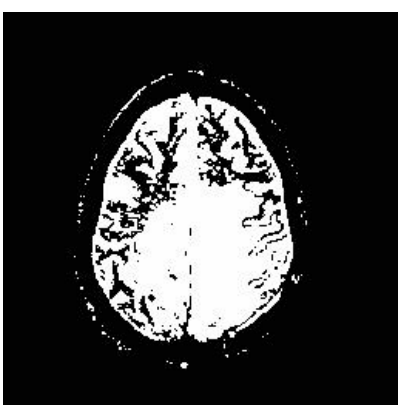

(b)

Figure 5. (a) Image after Otsu threshold, (b) Image after fuzzy c-means cluster.

\subsection{Digital Watermarking}

For embedding and extracting a watermark, we used DWT and IDWT. This process was used due to its robustness, better quality, and localization [21]. Robust digital watermarking is used to ensure good quality of the original image even after different attacks [22]. In DWT, a two-dimensional image is decomposed into four sub-bands. Low-Low (LL) is the top left sub-band that has the low frequency of the image, Low-High (LH) is the bottom right sub-band that has the vertical details of the image, High-Low (HL) is the top right sub-band that has the horizontal details of the image and High-High $(\mathrm{HH})$ is the bottom right sub-band that has the high frequency of the image.

In the proposed model, after computing the first level DWT we chose the LL1 sub-band because it contains the most information of the original image [4]. After taking the LL band, we computed the second level DWT in the LL1 and sub-band and choose LL2 for more robustness. Then, the watermark was embedded into the LL2 sub-band. The structure of the second level DWT is shown in Figure 6a and the second level DWT of the host image is presented in Figure $6 b$, where the watermark is implanted in low-frequency coefficient of the host image that contains most of the information. As a result, the image is less vulnerable against various attacks.

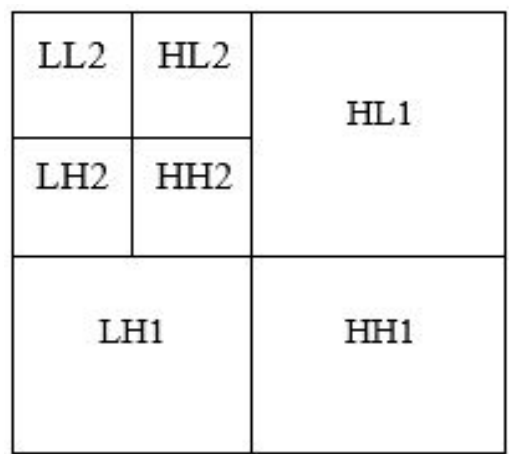

(a)

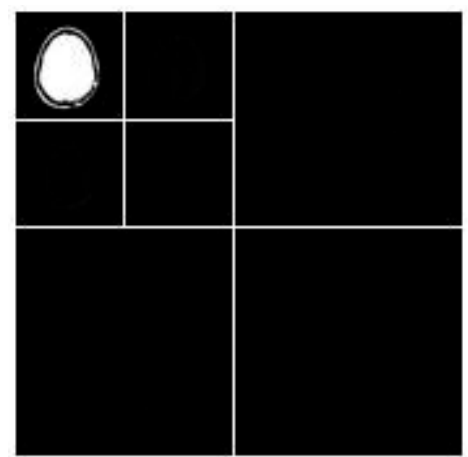

(b)

Figure 6. (a) Structure of second level DWT, (b) Second level DWT of the image.

\section{Experimental Results}

For evaluating the robustness and imperceptibility of the proposed model, we set up an experiment using a personal computer (PC) with the following configurations: AMD FX(tm)-8300 Eight-Core Processor, $\sim 3.3 \mathrm{GHz}, 16384 \mathrm{MB}$ RAM. As a simulation tool, we utilize MATLAB software version R2016a. To evaluate the proposed model, we used the Computer Vision Group, University of Granada (CVG UGR) Image dataset, where all of the images are $256 \times 256$ in size. As a watermark image, we used an image of size $512 \times 512$, which is presented in Figure $7 \mathrm{~b}$. To keep the uniform size of the input image and watermark image, we converted both images to $2048 \times 2048$. The watermark image 
was embedded into the MR host image. Figure 7 shows a sample of the host image, watermark, and watermarked image.

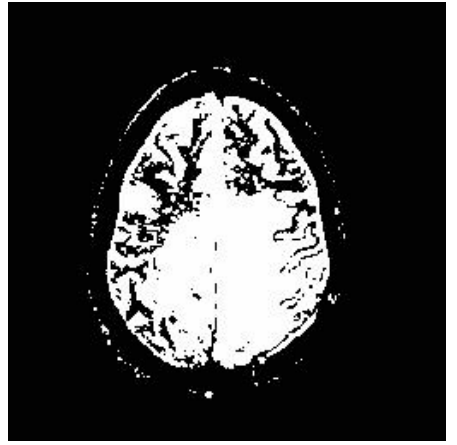

(a)

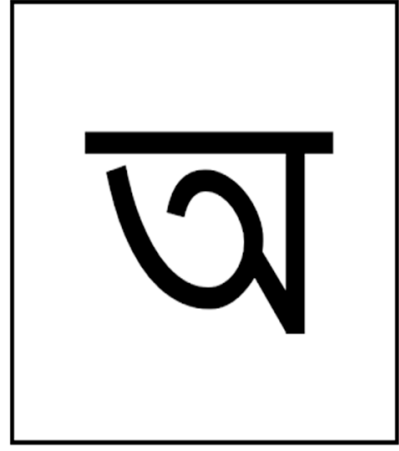

(b)

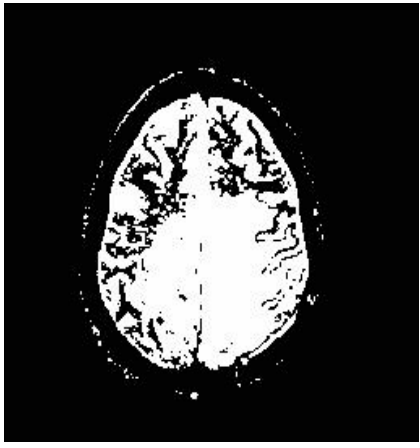

(c)

Figure 7. (a) Image after segmentation, (b) Watermark image and (c) Watermarked image.

After the embedding process, the watermarked image was passed through different attacks. Then, the watermark image was extracted from the watermarked image. The extraction process was performed by IDWT [23], which is the reverse process of DWT. After completion of the extraction process, the performance was evaluated by comparing the extracted watermark image and the original watermark image.

The performance (in terms of robustness and imperceptibility) of our model was evaluated with the following parameters: PSNR, BER, CC, and SSIM, where PSNR was used for imperceptibility, and BER and CC were used for robustness. SSIM was used to measure the similarity between the image before and after extraction. PSNR measured the amount of distortion of the watermarked image. In general, the greater value of PSNR represents less distortion.

$$
\text { PSNR }=10 \log _{10}\left(\frac{\text { peakval }^{2}}{\sqrt{\frac{\sum_{x=1}^{M} \sum_{y=1}^{N}\left\{W(x, y)-W^{\prime}(x, y)\right\}^{2}}{M * N}}}\right) .
$$

For Equations (1) and (2), $M$ and $N$ are the number of rows and columns, respectively, $\mathrm{w}$ and $\mathrm{w}^{\prime}$ represent the original image and extracted image, respectively, and $x$ and $y$ denote the pixel location. Length of the watermark image is expressed by L [24]. Pixel values can be positive or negative. Due to this fact, the summation is squared to avoid the issue where positive and negative values can cancel out each other. Then, the mean value is the square rooted for equality. On the other hand, peakval represents the maximum possible pixel value of the original image. When each pixel is denoted by 8 bits, the peakval will be 255 . For instance, if two images are identical to each other, then the divisor value is zero, which is eventually converted to a PSNR value of infinity.

BER evaluates the transmission accuracy where the value closer to zero illustrates a higher quality of image.

$$
B E R=\frac{\sum_{x=1}^{M} \sum_{y=1}^{N}\left|w^{\prime}(x, y)-w(x, y)\right|}{L} .
$$

The CC is calculated using Equation (3). A CC value near to one illustrates better robustness.

$$
\rho(A, B)=\frac{1}{N-1} \sum_{i=1}^{N}\left(\overline{\frac{A_{i}-\mu_{A}}{\sigma_{A}}}\right)\left(\frac{B_{i}-\mu_{B}}{\sigma_{B}}\right) .
$$


The SSIM measures the similarity between the original image and the extracted image.

$$
\operatorname{SSIM}=\frac{\sum_{x=1}^{M} \sum_{y=1}^{N}\left\{W(x, y) * W^{\prime}(x, y)\right\}}{\sum_{x=1}^{M} \sum_{y=1}^{N} W(x, y)^{2} * \sum_{x=1}^{M} \sum_{y=1}^{N} W^{\prime}(x, y)^{2}} .
$$

To evaluate the effectiveness, imperceptibility, and robustness, the result of the proposed model was compared with the other state-of-the-art watermarking models. Tables 1-4 present the evaluation results and comparison. Table 1 illustrates the PSNR values of the proposed model along with existing models after extraction of the watermark that had passed through different attacks such as the Salt and Pepper, Median Attack, Shear Attack, Blur Attack, Contrast Adjustment Attack, and Average Attack or without attack. The PSNR values of the other models are less than the PSNR values of the proposed model, which indicates that the proposed model is superior to others in terms of imperceptibility and distortion. Table 1 illustrates the PSNR values of the watermark image of different models including our proposed model after extracting the watermark from the host image, which clearly indicates that the proposed model has higher PSNR values compared to other models.

Table 1. Peak signal-to-noise ratio (PSNR) values of different models and the proposed model after extraction of the watermark.

\begin{tabular}{cccccc}
\hline Attacks & DWT & PSO-DWT & SVD-SWT & DCT-DWT & $\begin{array}{c}\text { Proposed } \\
\text { Model }\end{array}$ \\
\hline Without Attack & 40.108059 & 32.66059 & 41.957983 & 42.033778 & 42.140669 \\
Salt and Pepper & 44.166558 & 37.125007 & 28.742353 & 10.092619 & 43.127117 \\
Median & 42.585024 & 43.937647 & 31.415702 & 22.23475 & 51.721594 \\
Shear & 34.130048 & 11.020953 & 2.575604 & 19.689004 & 21.623157 \\
Blur & 47.791597 & 31.063986 & 19.923953 & 18.70545 & 50.712338 \\
Contrast Adjustment & 22.060379 & 29.604466 & 58.46861 & 26.743678 & 33.096049 \\
Average & 43.34289 & 30.828611 & 27.850806 & 20.158203 & 49.301436 \\
\hline
\end{tabular}

Table 2 exhibits the BER values of the proposed model along with other models with and without different attacks such as the Median Attack, Shear Attack, Average Attack, Blur Attack, and Gamma Correction attack. BER suggests the bit-wise error rate. Usually, lower values of BER indicate better image quality. As depicted in Table 2, BER values of the other models are much greater than the BER values of the proposed model for different attacks. This indicates that the proposed model is superior to others in terms of robustness and distortion.

Table 2. Bit error rate (BER) values of different models and the proposed model after extracting the watermark.

\begin{tabular}{ccccccc}
\hline Attacks & DWT & FCM-DWT & PSO-DWT & SVD-SWT & DCT-DWT & $\begin{array}{c}\text { Proposed } \\
\text { Model }\end{array}$ \\
\hline Without Attack & 0.05030 & 0.053559 & 0.424059 & 0.072922 & 0.045898 & 0.037152 \\
Median & 0.05055 & 0.060419 & 0.478366 & 0.082062 & 0.094727 & 0.041833 \\
Shear & 0.55753 & 0.527414 & 0.737429 & 0.698776 & 0.114258 & 0.524626 \\
Average & 0.05598 & 0.075205 & 0.537355 & 0.090286 & 0.112305 & 0.053675 \\
Blur & 0.09947 & 0.099012 & 0.574958 & 0.116898 & 0.125977 & 0.071027 \\
Gamma Correction & 0.08033 & 0.074181 & 0.36595 & 0.086029 & 0.06543 & 0.04863 \\
\hline
\end{tabular}

Table 3 exhibits the values of CC of the proposed model along with other state-of-the-art models with and without different attacks such as the Salt and Pepper, Median Attack, Average Attack, Gaussian Noise Attack, Speckle Attack, and Poison attack. CC values close to 1 suggest that the image has better quality. In Table 3 the CC values of our proposed model are much closer to 1 compared with the other models. 
Table 3. Cross-correlation (CC) values of different models and the proposed model after extracting the watermark.

\begin{tabular}{cccccc}
\hline Attacks & DWT & FCM-DWT & PSO-DWT & DCT-DWT & Proposed Model \\
\hline Without Attack & 0.715358 & 0.684247 & 0.216494 & 0.707538 & 0.73562 \\
Salt and Pepper & 0.142956 & 0.177559 & 0.10676 & 0.166058 & 0.190398 \\
Median & 0.613023 & 0.550206 & 0.046645 & 0.480141 & 0.633066 \\
Average & 0.659177 & 0.341573 & 0.019008 & 0.396111 & 0.40046 \\
Gaussian Noise & 0.281294 & 0.322411 & 0.208801 & 0.119289 & 0.338707 \\
Speckle & 0.110913 & 0.334645 & 0.202961 & 0.309736 & 0.352799 \\
Poison & 0.190138 & 0.346266 & 0.217656 & 0.134479 & 0.369062 \\
\hline
\end{tabular}

Table 4 exhibits the values of SSIM of the DWT model, FCM-DWT model, PSO-DWT model, SWT-SVD model, DCT-DWT model, and our proposed model after passing through different attacks such as the Median Attack, Shear attack, Average Attack, Blur Attack, Gamma Correction Attack, and Rotation Attack or without any attacks. SSIM suggests the similarity between the two images (original watermark and extracted watermark image). Greater values of SSIM represent more similarity between two images. As presented in Table 4, the SSIM values of the proposed model are greater than the other models after passing through different attacks.

Table 4. Structural similarity index measures (SSIM) values of other models and the proposed model after extraction.

\begin{tabular}{ccccccc}
\hline Attacks & DWT & FCM-DWT & PSO-DWT & SVD-SWT & DCT-DWT & Proposed Model \\
\hline Without Attack & 0.559483 & 0.667909 & 0.066096 & 0.302382 & 0.653441 & 0.729581 \\
Median & 0.559457 & 0.667686 & 0.066001 & 0.145907 & 0.156568 & 0.729484 \\
Shear & 0.201288 & 0.231616 & 0.048515 & 0.003994 & 0.085817 & 0.255911 \\
Average & 0.559196 & 0.643121 & 0.065871 & 0.108153 & 0.104532 & 0.705016 \\
Blur & 0.557797 & 0.667199 & 0.065721 & 0.078742 & 0.115184 & 0.729356 \\
Gamma Correction & 0.053569 & 0.104087 & 0.065849 & 0.822022 & 0.349381 & 0.189104 \\
Rotation Attack & 0.278796 & 0.369123 & 0.065494 & 0.220958 & 0.019174 & 0.396623 \\
\hline
\end{tabular}

\section{Conclusions}

In this paper, an approach to digital watermarking on biomedical images was put forward to ensure the safety and confidentiality of the image. This process is an application of biometrics since it provides unique identification through retention of a watermark after transmission. Additionally, access to the medical images by the authorized users can be validated through cross checking of the distinct watermark. The proposed model utilizes the improved SMQT, Otsu threshold, fuzzy c-means, DWT, and IDWT. Firstly, the model operates on SMQT and Otsu threshold to pre-process the image. Secondly, the enhanced image is segmented using the Otsu threshold and fuzzy c-means. Then, the segmented image is watermarked using DWT and IDWT. After that, the watermark is extracted using the reverse process of embedding. Finally, the performance was measured, which indicates that the suggested scheme shows superior results in terms of efficiency and imperceptibility as well as upholding the robustness against various attacks.

Author Contributions: All authors contributed equally to the conception of the idea, the design of experiments, the analysis and interpretation of results, and the writing and improvement of the manuscript.

Funding: This work was supported by Electronics and Telecommunications Research Institute (ETRI) grant funded by the Korean government foundation (19ZS1320, The development of smart context-awareness technique for major industry acceleration).

Conflicts of Interest: The authors declare no conflicts of interest.

\section{References}

1. Lisowiec, A.; Nowakowski, A.; Ksiazek, L. Signal processing in switchgear e-diagnosis network. In Proceedings of the International Symposium on Power Electronics, Electrical Drives, Automation and Motion; Sorrento, Italy, 20-22 June 2012, pp. 993-996. [CrossRef] 
2. Gunsel, B.; Uludag, U.; Tekalp, A.M. Robust watermarking of fingerprint images. Pattern Recognit. 2002, 35, 2739-2747. [CrossRef]

3. Low, C.Y.; Teoh, A.B.J.; Tee, C. Fusion of LSB and DWT biometric watermarking for offline handwritten signature. In Proceedings of the 2008 Congress on Image and Signal Processing, Sanya, Hainan, China, 27-30 May 2008; pp. 702-708.

4. Goel, A. Discrete Wavelet Transform (DWT) with Two Channel Filter Bank and Decoding in Image Texture Analysis. Int. J. Sci. Res. 2014, 3, 391-397.

5. Rathi, N.; Holi, G. Securing Biomedical Images by Watermarking Using DWT-DCT-SVD. arXiv 2014, arXiv:1406.7226.

6. Mehto, A.; Mehra, N. Adaptive Lossless Biomedical Image Watermarking Algorithm Based on DCT \& DWT. Procedia Comput. Sci. 2016, 78, 88-94.

7. Gaata, M.; Puech, W.; Sadkhn, S. Digital Watermarking Method based on Fuzzy Image Segmentation Technique. In Proceedings of the IEEE International Symposium on Signal Processing and Information Technology (ISSPIT 2011), Bilbao, Spain, 14-17 December 2011.

8. Kumar, V.; Lautan, R.; Faisal, M.; Pandey, K.M. DWT and Particle Swarm Optimization Based Digital Image Watermarking. Int. J. Eng. Res. Technol. 2013, 2, 2278-2281.

9. Kumar, S.B.; Ramashri, T. Robust SWT SVD Based Digital Image Watermarking Technique. Int. J. Comput. Sci. Inf. Eng. Technol. 2012, 2,1-4.

10. Mahmood, A.; Obimbo, C.; Hamed, T.; Dony, R. Improving the Security of the Biomedical Images. Int. J. Adv. Compu. Sci. Appl. 2013, 4, 137-146.

11. Eswaraiah, R.; Reddy, E. Biomedical Image Watermarking Technique for Accurate Tamper Detection in ROI and Exact Recovery of ROI. Int. J. Telemed. Appl. 2014, 2014, 13. [CrossRef]

12. Shukla, A.; Singh, C. Medical Image Authentication Through Watermarking. Int. J. Adv. Res. Comput. Sci. Technol. 2014, 2, 292-295.

13. Islam, M.; Kim, C.; Kim, J. A GPU-Based $(8,4)$ Hamming decoder for secure transmission of watermarked biomedical images. Clust. Comput. 2014, 18, 333-341. [CrossRef]

14. Aparna, P.; Kishore, P. An Efficient Biomedical Image Watermarking Technique in E-Healthcare Application Using Hybridization of Compression and Cryptography Algorithm. Int. J. Intell. Syst. 2018, 27, 115-133.

15. Ma, J.; Xou, C.; Jin, X. An Improved Image Enhancement Algorithm. Wuhan Univ. J. Nat. Sci. 2017, 22, 85-92. [CrossRef]

16. Shapiro, L.; Stockman, G. Computer Vision., 1st ed.; Personal Prentice Hall: Upper Saddle River, NJ, USA, 2001; pp. 279-325.

17. Otsu, N. A threshold selection method from gray-level histogram. IEEE Trans. Syst. Man, Cybern. 1979, 9, 62-66. [CrossRef]

18. Liao, P.S.; Chen, T.S.; Chung, P.C. A fast algorithm for multilevel thresholding. J. Inf. Sci. Eng. 2003, 17, 713-727.

19. Izakian, H.; Abraham, A.; Snášel, V. Fuzzy clustering using hybrid fuzzy c-means and fuzzy particle swarm optimization. In Proceedings of the 2009 World Congress on Nature \& Biologically Inspired Computing (NaBIC 2009), Coimbatore, India, 9-11 December 2009. [CrossRef]

20. Bazdek, J.C. Fuzzy Mathematics in Pattern Classification. Ph.D. Thesis, Cornel University, Ithaca, NY, USA, 1974.

21. Krishna, D.; Sarvanan, D. A survey on Digital Image Watermarking. Int. Refereed J. Eng. Sci. 2014, 1, 49-53.

22. Loukhaoukha, K.; Chouinard, J.Y.; Taieb, M.H. Optimal Image Watermarking Algorithm Based on LWT-SVD via Multi-objective Ant Colony Optimization. J. Inf. Hiding Multimed. Signal Process. 2011, 2, 303-319.

23. Ingale, S.P.; Dhote, C.A. Digital Watermarking Algorithm using DWT Technique. Int. J. Comput. Sci. Mob. Comput. 2016, 5, 1-9.

24. Yang, F.F.; Wang, C.Y.; Huang, W.; Zhou, X. Embedding Binary Image Watermark in DC Components of All Phase Discrete Cosine Biorthogonal Transform. Int. J. Secur. Its Appl. 2015, 9, 125-136. [CrossRef]

(C) 2019 by the authors. Licensee MDPI, Basel, Switzerland. This article is an open access article distributed under the terms and conditions of the Creative Commons Attribution (CC BY) license (http://creativecommons.org/licenses/by/4.0/). 